\title{
Report
}

\section{Band 3 Protein Function and Oxidative Stress in Erythrocytes from Systemic Sclerosis Patients with Interstitial Lung Disease}

\author{
Rossana Morabito $^{1}$, Alessia Remigante ${ }^{1}$, Gianluca Bagnato ${ }^{2}$, William Neal Roberts ${ }^{3}$, \\ Davide Sciortino', Tommaso D'Angelo $^{4}$, Filippo Iannelli ${ }^{2}$, Dario Iannelli ${ }^{2}$, Francesca Cordova ${ }^{2}$, \\ Mariateresa Cirillo ${ }^{2}$, Giuseppina La Spada ${ }^{1}$, Angela Marino ${ }^{1,}$, \\ ${ }^{1}$ Department Chemical, Biological, Pharmaceutical and Environmental Sciences, University of Messina, Messina, Italy \\ ${ }^{2}$ Department Clinical and Experimental Medicine, University of Messina, Messina, Italy \\ ${ }^{3}$ Department Medicine, University of Louisville, Kentucky, US \\ ${ }^{4}$ Department Biomedical Sciences and Morphological and Functional Imaging, University of Messina, Messina, Italy
}

\author{
Email address: \\ marinoa@unime.it (A. Marino) \\ ${ }^{*}$ Corresponding author
}

\section{To cite this article:}

Rossana Morabito, Alessia Remigante, Gianluca Bagnato, William Neal Roberts, Davide Sciortino, Tommaso D’Angelo, Filippo Iannelli, Dario Iannelli, Francesca Cordova, Mariateresa Cirillo, Giuseppina La Spada, Angela Marino. Band 3 Protein Function and Oxidative Stress in Erythrocytes from Systemic Sclerosis Patients with Interstitial Lung Disease. European Journal of Clinical and Biomedical Sciences.

Vol. 3, No. 4, 2017, pp. 80-84. doi: 10.11648/j.ejcbs.20170304.12

Received: May 16, 2017; Accepted: June 28, 2017; Published: July 27, 2017

\begin{abstract}
A link between reactive oxygen species (ROS) and Systemic Sclerosis (SSc) development has been already described, along with an oxidative stress-induced reduction of anion exchange capability through Band 3 protein. On this basis, blood from SSc patients has been collected to measure the rate constant for $\mathrm{SO}_{4}{ }^{*}$ uptake, which is an index for anion exchange capability through Band 3 protein. This parameter was significantly reduced in SSc patients, with or without Interstitial Lung Disease, a complication of SSc. As a reduced Band 3 protein function was observed in SSc patients with either low or high levels of malonildialdehyde (MDA), the end product of lipoperoxidation, anion exchange through erythrocytes membrane may be not univocally related to oxidative stress and, what is more, can be considered as a sensitive tool to monitor SSc development.
\end{abstract}

Keywords: Band 3 Protein, Erythrocytes, Interstitial Lung Disease, Oxidative Stress, Systemic Sclerosis

\section{Introduction}

The role of oxidative stress in pathogenesis diseases has risen much more attention in the recent years, with specific regard to diabetes [1], aged-related pathologies including neurodegenerative diseases [2-4] and, most recently, canine leishmaniasis [5]. Systemic Sclerosis (SSc), an autoimmune connective tissue disorder, has been chosen as subject of the present report. SSc is associated with many different clinical patterns, at tissue and internal organ levels, possibly mediated by progressive fibrosis. Skin lesions and microvascular alterations are also described. Though its pathophysiology is still unclear, a link between reactive oxygen species (ROS) and SSc development has been ascertained [6]. In particular, high ROS levels in patients with diffuse cutaneous SSc (dcSSc) have been associated with increased levels of oxidative stress markers, alteration of antioxidant mechanisms, reduced non-enzymatic antioxidants, such as ascorbic acid, $\alpha$ tocopherol, $\beta$-carotene and selenium and reduced antioxidant defense [7-10]. The link between oxidative stress and SSc has been further supported by a mouse model exhibiting a disease closely resembling human SSc when administered hypochlorous acid, as oxidative agent [11].

This background motivated the focus on SSc and its complications and led to monitor Band 3 protein efficiency in erythrocytes of SSc patients displaying pulmonary fibrosis as a complication of such disease. 
Band 3 protein is the most abundant integral membrane protein of erythrocytes $[12,13]$, responsible for $\mathrm{Cl}^{-} / \mathrm{HCO}_{3}{ }^{-}$ exchange, which correlates with ion balance across membrane osmotic properties, cell shape maintaining, gas exchange and, in turn, oxygenation of the entire organism [14]. These functions are mediated by both membrane and cytoplasmic domains via phosphorylation pathways, as recently shown [15, 16]. Other studies have demonstrated a reversible interaction of Band 3 protein with hemoglobin and spectrin [17, 18], suggesting that the efficiency of Band 3 protein reflects whole erythrocyte homeostasis.

$\mathrm{SO}_{4}{ }^{=}$transport measurement, as more easily detected than $\mathrm{Cl}^{-}$or $\mathrm{HCO}_{3}^{-}$uptake [12], significantly accounts for $\mathrm{Cl}^{-} / \mathrm{HCO}_{3}{ }^{-}$exchange and can be monitored by a turbidimetric method [12-19] based on measurement of the rate constant for $\mathrm{SO}_{4}{ }^{=}$uptake. This method, enabling determination of anion exchange velocity, has been recently confirmed as a good tool to verify the impact of oxidative stress on human erythrocytes exposed to xenobiotics, natural toxins or hydrogen peroxide $[19,20]$. With regard to this latter point, the authors [19] demonstrated that ion transport through erythrocytes membrane is a sensitive tool to detect oxidant effects provoked by hydrogen peroxide at concentrations within a physiological range (under $1 \mathrm{mM}$ ) and not eliciting production of Malonildialdehyde (MDA). MDA is the end product of lipid peroxidation, and hence considered as an index of lipoperoxidation.

Based on this evidence, the aim of the present report was to pursue this novel issue in rheumatology by checking anion exchange capability through Band 3 protein in erythrocytes from SSc patients with pulmonary fibrosis, recruited in Southern Italy (Sicily). Patients gave their informed consent prior to their inclusion in the protocol which was carried out in accordance with the Declaration of Helsinki and Good Clinical Practice.

Blood samples were withdrawn from 15 healthy patients and $8 \mathrm{SSc}$ patients with no HRTC (High resolution CT) sign of pulmonary fibrosis. Nine SSc patients (6 females and 3 males) were affected by interstitial lung disease (SSc-ILD). SSc patients with no HRTC signs of pulmonary fibrosis had $51.2 \pm$ 9.4 years of age, while those with ILD had $53.2 \pm 7.6$ years of age. All patients were affected by the diffuse form of disease. Disease duration, considered as the time since the first non-Raynaud symptom, was $3.5 \pm 2$ years for patients with ILD and $4.1 \pm 2.3$ for SSc patients with no HRTC sign of pulmonary fibrosis.

Erythrocytes were suspended to $3 \%$ hematocrit in isotonic medium, containing $116 \mathrm{mM} \mathrm{Na}_{2} \mathrm{SO}_{4}, 10 \mathrm{mM}$ HEPES, $15 \mathrm{mM}$ glucose, $\mathrm{pH} 7.4$, osmotic pressure $300 \mathrm{mOsm}$. Levels of $\mathrm{SO}_{4}{ }^{=}$ were spectrophotometrically read at $425 \mathrm{~nm}$ wavelength, and the absorption was converted to millimolar of intracellular $\mathrm{SO}_{4}{ }^{=}$using a calibrated standard curve previously obtained by precipitating known $\mathrm{SO}_{4}{ }^{=}$concentrations $\left(\mathrm{mg} / \mathrm{L}\right.$ cells $\left.10^{-2}\right)$. $\left[\mathrm{SO}_{4}{ }^{=}\right] \mathrm{L}$ cells $10^{-2}$ reported in graphs represent $\mathrm{SO}_{4}{ }^{=}$ concentration trapped by $10 \mathrm{~mL}$ erythrocytes suspended at $3 \%$ hematocrit. Intracellular $\mathrm{SO}_{4}{ }^{2}$, determined at different time intervals (5-10-15-30-45-60-90-120 $\mathrm{min}$ ), was considered to calculate the rate constant $\left(\mathrm{min}^{-1}\right)$ [18]. Reciprocal of the rate constant for $\mathrm{SO}_{4}{ }^{=}$uptake $(\mathrm{min})$ represents time needed to reach $63 \%$ of the total $\mathrm{SO}_{4}{ }^{2}$ intracellular concentration. Once ascertained Band 3 protein efficiency in anion exchange on SSc patients with or without ILD, MDA plasma levels, along with intracellular content of Reduced Glutathione (GSH) and Methemoglobin (MetHb), were measured to determine oxidative conditions, in SSc patients exhibiting ILD.

\section{Results and Discussion}

Figure 1A shows the time course for $\mathrm{SO}_{4}{ }^{2}$ uptake in control conditions (healthy patients) and in SSc patients with or without ILD. $\mathrm{SO}_{4}=$ transport in control erythrocytes increased steeply at the initial stage and reached equilibrium in $30 \mathrm{~min}$, exhibiting a rate constant of $0.053 \pm 0.001 \mathrm{~min}^{-1}$.

The rate constant for $\mathrm{SO}_{4}{ }^{=}$uptake measured in erythrocytes from SSc patients with $\left(0.035 \pm 0.002 \mathrm{~min}^{-1}\right)$ or without ILD $\left(0.037 \pm 0.001 \mathrm{~min}^{-1}\right)$ was significantly lower than in control erythrocytes and $\mathrm{SO}_{4}{ }^{=}$quantity trapped by the cells was significantly reduced as well. The reduction in both rate constant for $\mathrm{SO}_{4}{ }^{=}$uptake and internalized $\mathrm{SO}_{4}{ }^{=}$have been correlated to oxidative conditions detected in blood. Afterwards, only SSc-ILD patients were considered to complete the experimental plan. In this latter regard, in a separate experimental set, oxidative parameters have been measured and analyzed by one way ANOVA followed by Bonferroni's post hoc test: MetHb (figure 1B) levels in females and males with pulmonary fibrosis (SSc-ILD) were significantly different with respect to control, with levels in females significantly higher than in males. GSH levels (figure $1 C)$ in both SSc-ILD female and male patients were significantly lower than control. MDA plasma levels (figure 1D), measured in both SSc-ILD female and male patients, were significantly higher than control, with MDA levels in males significantly higher than in females.

The present data show that erythrocytes from SSc patients exhibit a reduction in both anion exchange capability through Band 3 protein and in $\mathrm{SO}_{4}{ }^{=}$content internalized by the cells during the observation time, with respect to erythrocytes from healthy patients. The extent of such decrease is comparable to that one recently observed in erythrocytes collected from healthy patients and then treated with hydrogen peroxide [19, 20]. In particular, the authors demonstrated that oxidative conditions, modeled by hydrogen peroxide within physiological range of concentrations (not higher than 300 $\mu \mathrm{M}$ ), compromises anion exchange efficiency, though not inducing lipid peroxidation, thus demonstrating the sensitivity of the method (rate constant for $\mathrm{SO}_{4}{ }^{=}$uptake measurement) to assess erythrocytes homeostasis. Hence, this method has been here used to monitor the impact of oxidative stress on SSc development.

The evidence that alterations in anion exchange capability through Band 3 protein may account for disease development has been recently provided [5]. The authors demonstrated that in erythrocytes collected from dogs with leishmaniasis, an oxidative stress-related disease, the rate constant for $\mathrm{SO}_{4}{ }^{=}$ 
uptake is significantly reduced with respect to control. Such evidence further corroborates the hypothesis that anion exchange through Band 3 protein measurement may account for development of oxidative stress-related pathologies and for homeostasis of the whole organism. In this respect, it is already known that Band 3 protein function is critically related
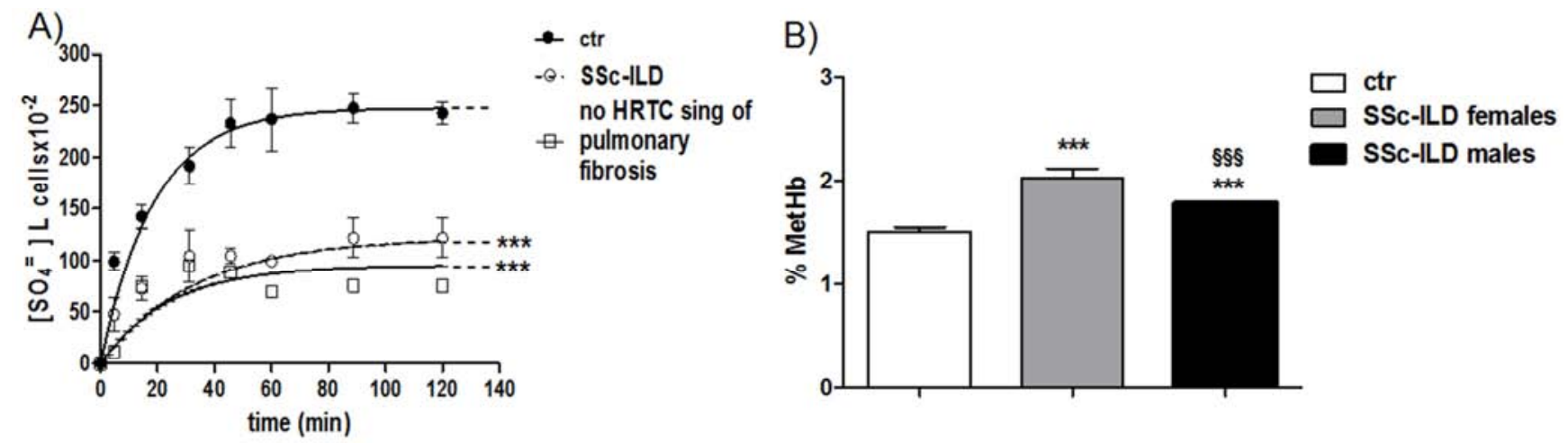

not only to anion balance, but also to oxygenation state of erythrocytes and, in turn, of the entire animal. In this scenario, Band 3 protein function assessment may also reasonably account for oxygenation state of SSc patients, namely those affected by Interstitial Lung Disease, a frequent complication of SSc development.
C)

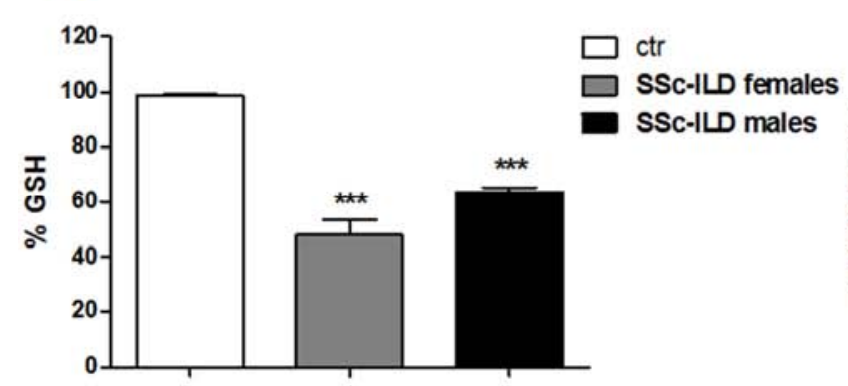

Figure 1. (A) Time course of $\mathrm{SO}_{4}{ }^{=}$uptake measured in erythrocytes from both healthy (control, $N=15$ ) and $S S c$ patients $(N=6$ for female samples (F), $N=3$ for male samples $(M))$. Points represent the mean \pm SEM from separate experiments, where ***p<0.001 significant different versus control. (B) MetHb levels in $F$ and $M$ patients with SSc-ILD were significantly different with respect to control (***p<0.001), with levels in $F$ statistically higher than in $M(\$ \$ \$ p<0.001)$. (C) GSH levels and MDA levels in both SSc-ILD F and M patients were significantly lower than control $(* * * p<0.001)$. (D) MDA plasma levels measured in both $F$ and M SSC-ILC patients. MDA levels in M were significantly higher than in F patients $\$ \$ p<0.001)$.

Band 3 protein efficiency is reportedly known to account for erythrocytes deformability [12] an essential condition for blood flow. As vascular damage compromising microcirculation and leading, in turn, to ischemic episodes in SSc patients has been previously described, Band 3 protein function monitoring may be reasonably proposed as a potentially useful tool to verify erythrocytes behavior alterations during disease progression. Alterations at level ofstructural membrane properties, possibly revealed by Band 3 protein changes, may in fact lead to intravascular alterations occurring during SSc, contributing to blood hyperviscosity and exacerbating local ischemia [21]. More specifically, other authors [22] already reported structural alterations of Band 3 protein in SSc patients. In particular, reduced Band 3 protein expression levels correlating with phosphatidylserine externalization and, in turn, with eryptosis, has been ascertained.

The present report adds novel elements in this regard, as it has been recently demonstrated that progression of oxidative stress-related pathologies, such as canine leishmaniasis [5], may not be associated with a reduction in Band 3 protein expression levels, but still associated to a reduced anion exchange capability through this membrane protein. Therefore, measurement of the rate constant for $\mathrm{SO}_{4}{ }^{=}$uptake may be considered as a more sensitive tool to monitor $\mathrm{SSc}$ development, and could be added to laboratory testing currently performed in clinical practice.

As a whole, the present findings may suggest a strong correlation between Band 3 protein efficiency in SSc patients and oxidative stress, supported by the evidence that levels of $\mathrm{MetHb}$ and MDA are significantly augmented in SSc patients, while GSH intracellular content is reduced. Band 3 protein in SSc patients, with or without ILD, is affected to the same extent, in spite of different MDA plasma levels, which confirms the sensitivity of the method. Furthermore, SSc development seems to be associated with destabilization of membrane protein arrangement, revealed by a reduced efficiency in anion exchange not dependent on 
lipoperoxidation, which is in line with what already proven in human erythrocytes exposed to hydrogen peroxide [19].

With regard to rate constant for $\mathrm{SO}_{4}=$ uptake determination (Figure 1A), sex differences have not been considered, due to samples availability. Nonetheless, as levels of MetHb and MDA (Figure 1B, D) were significantly different in male and female patients, a different response of erythrocytes to disease development according to sex differences should be hypothesized, thus contributing, in perspective, to gender medicine.

It is worthy of note that other authors have already correlated SSc development with oxidative stress by conducting their experiments on skin obtained from SSc patients [6]. The present report provides more knowledge about SSc development by monitoring erythrocytes homeostasis and proposing Band 3 protein anion exchange capability as a tool to fulfill the aim of, on one hand, verifying disease progression and, on the other hand, facilitating future investigations. Investigators have been hampered in the enrollment of patients to study skin fibrosis during SSc development [6]. Therefore, the use of the method proposed by the present report, being Band 3 anion exchange monitoring performed on aliquots of blood samples routinely withdrawn from patients, may serve as a biomarker or even a surrogate marker allowing easier study of patients with shorter disease duration.

One more novel aspect arising from the present report is disease duration. The study has been performed on patients with an average disease duration of 3.5 years, considered as the time from the first non-Raynaud symptom, which is shorter than what reported elsewhere [6]. In this light, the study of Band 3 protein efficiency in anion exchanging may be useful in defining a suitable time window for pharmacological treatment before skin lesions extension is exacerbated by disease progression.

In the present report phosphorylation pathways mediating Band 3 protein function $[15,16]$ have not been considered. As a matter of fact, as oxidative conditions associated with canine leishmaniasis have been already demonstrated to alter Band 3 protein anion exchange capability via endogenous antioxidant system and phosphorylative pathways [5], an involvement of this signaling pathways in SSc development can not be excluded and may be the subject of future studies.

\section{Conclusions}

The present report confirms that oxidant/antioxidant imbalance affects erythrocytes of SSc patients and shows that: i) the rate constant for $\mathrm{SO}_{4}{ }^{=}$uptake measurement is useful in monitoring erythrocytes homeostasis in oxidative stress-related pathologies such as SSc, namely at a duration of disease comprised between 3 and 4 years; ii) Band 3 protein could be considered as a candidate for drug development, currently involving antioxidants compounds; iii) erythrocytes oxidative state may be considered as a good biomarker for studying development of SSc and its complications.

\section{References}

[1] Yang W, Fu J, Yu M, Wang D, Rong Y, Yao P, et al. Effects of three kinds of curcuminoids on anti-oxidative system and membrane deformation of human peripheral blood erythrocytes in high glucose levels. Cell Physiol Biochem.2015, 35:789802 .

[2] Macchi B, Di Paola R, Marino-Merlo F, Felice MR, Cuzzocrea $\mathrm{S}$, Mastino A. Inflammatory and cell death pathways in brain and peripheral blood in Parkinson's disease. CNS Neurol Disord Drug Targets. 2015; 14(3):313-24.

[3] Liochev SI. Reactive oxygen species and the free radical theory of aging. Free Radic Biol Med. 2013; 60:1-4.

[4] Zheng YP, Xu DH. Zn(II)-curcumin protects against hemorheological alterations, oxidative stress and liver injury in a rat model of acute alcoholism. Env Toxicol Pharmacol. 2014; $37: 729-37$.

[5] Morabito R, Remigante A, Cavallaro M, Taormina A, La Spada $\mathrm{G}$, Marino A. Anion exchange through band 3 protein in canine leishmaniasis at different stages of disease. Pflugers Arch. 2017 469(5-6):713-24.

[6] Bourji K, Meyer A, Chatelus E, Pincemail J, Pigatto E,

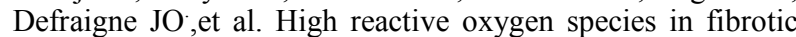
and nonfibrotic skin of patients with diffuse cutaneous systemic sclerosis. Free Radic Biol Med. 2015; 87:282-9. doi: 10.1016/j.freeradbiomed.2015.07.002.

[7] Ogawa F, Shimizu K, Muroi E, Hara T, Sato S. Increasing levels of serum antioxidant status, total antioxidant power, in systemic sclerosis. Clin Rheumatol. 2011; 30(7):921-5.

[8] Cruz-Domínguez MP, Montes-Cortes DH, Olivares-Corichi IM, Vera-Lastra O, Medina G, Jara LJ. Oxidative stress in Mexicans with diffuse cutaneous systemic sclerosis. Rheumatol Int. 2013; 33(9):2261-7.

[9] Tsou PS, Talia NN, Pinney AJ, Kendzicky A, Piera-Velazquez $\mathrm{S}$, Jimenez SA, et al. Effect of oxidative stress on protein tyrosine phosphatase 1B in scleroderma dermal fibroblasts. Arthritis Rheum. 2012; 64(6):1978-89. doi: 10.1002/art.34336.

[10] Fabrini R, Rosato E, Gigante A, Bocedi A, Cianci R, Barbano B, et al. Erythrocyte glutathione transferase: a non-antibody biomarker for systemic sclerosis, which correlates with severity and activity of the disease. Cell Death Dis. 2013; 4:e736. doi: 10.1038/cddis.2013.255.

[11] Bitto A, Bagnato GL, Pizzino G, Roberts WN, Irrera N, Minutoli L, et al. Simvastatin prevents vascular complications in the chronic reactive oxygen species murine model of systemic sclerosis. Free Radic Res. 2016; 50(5):514-22. doi: 10.3109/10715762.2016.1149171.

[12] Romano L, Passow H. Characterization of anion transport system in trout red blood cell. Am J Physiol. 1984; 246(3 Pt 1):C330-8.

[13] Reithmeier RAF, Casey JR, Kall AC, Sansomc MSP, Alguel Y, Iwata S. Band 3, the human red cell chloride/bicarbonate anion exchanger (AE1, SLC4A1), in a structural context. Biochim Biophys Acta. 2016; 1858:1507-32. 
[14] Chernyshova ES, Zaikina YS, Tsvetovskaya GA, Strokotov DI, Yurkin MA, Serebrennikova ES, et al. Influence of magnesium sulfate on $\mathrm{HCO}_{3} / \mathrm{Cl}$ transmembrane exchange rate in human erythrocytes. J Theor Biol. 2016; 393:194-202.

[15] Morabito R, Remigante A, Di Pietro ML, Giannetto A, La Spada G, Marino A. $\mathrm{SO}_{4}{ }^{=}$uptake and catalase role in preconditioning after $\mathrm{H}_{2} \mathrm{O}_{2}$-induced oxidative stress in human erythrocytes. Pflugers Arch. 2017; 469(2):235-250. doi: 10.1007/s00424-016-1927-1.

[16] Pantaleo A, Ferru E, Pau MC, Khadjavi A, Mandili G, Mattè A, et al. Band 3 erythrocyte membrane protein acts as redox stress sensor leading to its phosphorylation by p (72) Syk. Oxidative Med Cell Longev. 2016:6051093.

[17] Salhany JM, Sloan RL, Cordes KA. In situ cross-linking of human erythrocyte Band 3 by bis (sulfosuccinimidyl) suberate. Evidence for ligand modulation of two alternate quaternary forms: covalent band 3 dimers and noncovalent tetramers formed by the association of two covalent dimers. J Biol Chem. 1990; 265:17688-93.

[18] Franke RF, Scharnweber T, Fuhrmann R, Wenzel F, Kruger A, Mrowietz C, Jung F.Effect of radiographic contrast media on the spectrin/band 3-network of the membrane skeleton of erythrocytes. Plos One. 2014; 9(2):e89512.
[19] Morabito R, Romano O, La Spada G, Marino A. $\mathrm{H}_{2} \mathrm{O}_{2}$-induced oxidative stress affects $\mathrm{SO}_{4}{ }^{-}$transport in human erythrocytes. PLoS One. 2016; 11(1):e0146485. doi: 10.1371/journal.pone.0146485.

[20] Morabito R, Marino A, Romano P, Rigano C, La Spada G. Sulphate and chloride-dependent potassium transport in human erythrocytes are affected by crude venom from nematocysts of the jellyfish Pelagia noctiluca. Cell Physiol Biochem. 2013; 32:86-95.

[21] Solans R, Motta C, Solá R, La Ville AE, Lima J, Simeón P, et al Abnormalities of erythrocyte membrane fluidity, lipid composition, and lipid peroxidation in systemic sclerosis: evidence of free radical-mediated injury. Arthritis Rheum. 2000; 43(4):894-900.

[22] Giovannetti A, Gambardella L, Pietraforte D, Rosato E, Giammarioli AM, Salsano F, et al. Red blood cell alterations in systemic sclerosis: a pilot study. Cell Physiol Biochem. 2012; 30(2):418-27. 